\title{
Insulin Autoimmune Syndrome Caused by an Adhesive Skin Patch Containing Loxoprofen-Sodium
}

\author{
Satoko Okazaki-Sakai ${ }^{1,2}$, Sachiko Yoshimoto ${ }^{1}$, Kunimasa Yagi $^{2}$, Takanobu Wakasugi ${ }^{3}$, \\ Yoshiyu Takeda ${ }^{2}$ and Masakazu Yamagishi ${ }^{2}$
}

\begin{abstract}
A 62-year-old woman complained of repeated hypoglycemic events. A $75 \mathrm{~g}$ oral glucose tolerance test 75 gOGTT) showed a marked increase in the plasma insulin level and impaired glucose tolerance. The patient exhibited a high titer of plasma anti-insulin autoantibodies. Her diagnosis was insulin autoimmune syndrome (IAS). Following the cessation of loxoprofen-sodium (LOXs), she experienced no further hypoglycemic episodes. However, the hypoglycemic attacks recurred following the accidental readministration of LOXs in an adhesive skin patch. Considering the changes in the titer of anti-insulin autoantibodies, the repeated 75 gOGTT and the repeated Scatchard analysis, we determined LOXs to be the cause of the IAS and evaluated the characteristics of the autoantibodies.
\end{abstract}

Key words: insulin autoimmune syndrome, loxoprofen-sodium, adhesive skin patch, $75 \mathrm{~g}$ oral glucose tolerance test, Scatchard analysis

(Intern Med 52: 2447-2451, 2013)

(DOI: 10.2169/internalmedicine.52.0570)

\section{Case Report}

A 62-year-old woman visited our hospital in April 2009. Her symptoms included repeated faintness, hunger, sweating, palpitations and finger tremors before lunch time. On the patient's first visit, her blood glucose level was $62 \mathrm{mg} /$ $\mathrm{dL}$ and her symptoms disappeared quickly after receiving an intravenous glucose injection. At that time, the symptoms were suspected to reflect hypoglycemic episodes. A few days later, a $75 \mathrm{~g}$ oral glucose tolerance test (75gOGTT) showed a marked increase in the plasma insulin level and impaired glucose tolerance (Table 1: Onset of hypoglycemia). The plasma insulin level at 120 minutes was 2,995 $\mu \mathrm{U} / \mathrm{mL}$, which was more than 100 times the level observed at the time of the 75gOGTT before the administration of loxoprofen-sodium (LOXs) (Table 1: Before hypoglycemia). The patient's past medical history included appendicitis at 20 years of age and sudden onset of deafness at 50 years of age. She denied having a history of a diagnosis of diabetes or receiving insulin therapy in the past. She had taken only eperison hydrochloride, rebamipide and LOXs since February 2009.

Her physical findings were as follows: height: $152 \mathrm{~cm}$, weight: $64 \mathrm{~kg}$, body mass index (BMI): $28 \mathrm{~kg} / \mathrm{m}^{2}$, blood pressure: 130/80 $\mathrm{mmHg}$. No abnormal findings were observed in the chest, abdomen or extremities. The postprandial laboratory data obtained on another day were as follows: plasma glucose: $55 \mathrm{mg} / \mathrm{dL}, \mathrm{HbAlc}$ (NGSP): $5.5 \%$ (1), plasma C-peptide: $1.33 \mathrm{ng} / \mathrm{mL}$, plasma insulin: $242 \mu \mathrm{IU} / \mathrm{mL}$, plasma glucagon: $150 \mathrm{pg} / \mathrm{mL}$, total cholesterol: $275 \mathrm{mg} / \mathrm{dL}$, HDL-C: $75 \mathrm{mg} / \mathrm{dL}$, LDL-C: $75 \mathrm{mg} / \mathrm{dL}$, triglycerides: 176 $\mathrm{mg} / \mathrm{dL}$, cholinesterase: $538 \mathrm{IU} / \mathrm{L}$ (normal range: 185-431). The titer of anti-insulin autoantibodies was $90 \%(<7.0)$. A Scatchard plot analysis identified two classes of binding sites. One site had an affinity constant (K1) of $0.0422 \times 10^{8}$ $\mathrm{M}^{-1}$ and a binding capacity (B1) of $13.1 \times 10^{-8} \mathrm{M}$ (high affinity/low capacity site) and the other had an affinity constant (K2) of $0.00203 \times 10^{8} \mathrm{M}^{-1}$ and a binding capacity (B2) of $36.7 \times 10^{-8} \mathrm{M}$ (low affinity/high capacity site). The results of the Scatchard analysis matched the features of IAS; however, the diagnosis was not well grounded because the

${ }^{1}$ Department of Endocrinology and Metabolism, Komatsu Municipal Hospital, Japan, ${ }^{2}$ Department of Internal Medicine, Kanazawa University Graduate School of Medical Science, Japan and ${ }^{3}$ Department of Endocrinology and Metabolism, Fukui Prefectural Hospital, Japan Received for publication March 22, 2013; Accepted for publication June 17, 2013

Correspondence to Dr. Satoko Okazaki-Sakai, satoko_sakai_1207@yahoo.co.jp 
Table 1. 75g Oral Glucose Tolerance Tests and HbA1c (NGSP)

\begin{tabular}{crrrrrrrr}
\hline & \multicolumn{2}{c}{$\begin{array}{c}\text { Before } \\
\text { hypoglycemia }\end{array}$} & \multicolumn{2}{c}{$\begin{array}{c}\text { Onset of } \\
\text { hypoglycemia }\end{array}$} & \multicolumn{2}{c}{$\begin{array}{c}\text { One month after } \\
\text { cessation of LOXs }\end{array}$} & \multicolumn{2}{c}{$\begin{array}{c}\text { Relapse of } \\
\text { Hypoglycemia }\end{array}$} \\
\cline { 2 - 10 }$(\mathrm{min})$ & $\begin{array}{c}\text { PG } \\
(\mathrm{mg} / \mathrm{dL})\end{array}$ & $\begin{array}{c}\text { IRI } \\
(\mu \mathrm{IU} / \mathrm{mL})\end{array}$ & $\begin{array}{c}\text { PG } \\
(\mathrm{mg} / \mathrm{dL})\end{array}$ & $\begin{array}{c}\text { IRI } \\
(\mu \mathrm{IU} / \mathrm{mL})\end{array}$ & $\begin{array}{c}\text { PG } \\
(\mathrm{mg} / \mathrm{dL})\end{array}$ & $\begin{array}{c}\text { IRI } \\
(\mu \mathrm{IU} / \mathrm{mL})\end{array}$ & $\begin{array}{c}\text { PG } \\
(\mathrm{mg} / \mathrm{dL})\end{array}$ & $\begin{array}{c}\text { IRI } \\
(\mu \mathrm{IU} / \mathrm{mL})\end{array}$ \\
\hline 0 & 90 & 4.4 & 77 & 134.7 & 90 & 52.7 & 85 & 78.5 \\
30 & 119 & 25.6 & 150 & 225.9 & 163 & 224.0 & 182 & 177.0 \\
60 & 129 & 29.9 & 204 & 1785 & 173 & 336.0 & 173 & 236.0 \\
90 & & & 179 & 2375 & 130 & 335.0 & 165 & 285.0 \\
120 & 146 & 26.6 & 158 & 2995 & 126 & 320.0 & 161 & 303.0 \\
150 & & & 114 & 2970 & & & & \\
180 & & & 99 & 2669 & 96 & 213.0 & 113 & 299.0 \\
HbA1c & $5.1 \%$ & $5.5 \%$ & $5.5 \%$ & & $5.2 \%$ & \\
\hline
\end{tabular}

Before hypoglycemia (November, 2008): 75g OGTT showed impaired glucose tolerance, 5 months before the onset of hypoglycemia, and 2 months before LOXs. Onset of hypoglycemia (April, 2009):75g OGTT of shortly after her first visit showed significantly increased serum insulin and glucose levels. One month after 1st cessation of LOXs (June, 2009): Normal glucose tolerance and decreased levels of serum insulin levels were demonstrated. Relapse of Hypoglycemia (November, 2009): One month after adhesive patch form of LOXs use, several hypoglycemic episodes re-occurred, and her glucose tolerance deteriorated again.

In April 2009, the level of HbAlc was higher than before onset. And in November the level of $\mathrm{HbAlc}$ returned to before onset. It seems that the Insulin antibody causes the resistance against effect of insulin.

Table 2. Diurnal Profile of Plasma Glucose, Insulin and C-peptide Levels

\begin{tabular}{cccc}
\hline Time & Glucose $(\mathrm{mg} / \mathrm{dL})$ & Insulin $(\mu \mathrm{IU} / \mathrm{mL})$ & $\mathrm{CPR}(\mathrm{ng} / \mathrm{mL})$ \\
\hline 8:00(Fasting) & 83 & 120 & 1.83 \\
$10: 00$ & 122 & 762 & 7.49 \\
$12: 00$ & 78 & 228 & 3.48 \\
$14: 00$ & 112 & 385 & 6.89 \\
$18: 00$ & 67 & 111 & 1.29 \\
$20: 00$ & 125 & 797 & 8.39 \\
$22: 00$ & 82 & 232 & 3.34 \\
\hline
\end{tabular}

The levels of plasma glucose, insulin, C-peptide were determined before and 2-hours after each meal, and at bedtime in the beginning of May, 2009. The disproportion between the levels of plasma glucose, insulin and C-peptide seemed to indicate the existence of insulin antibodies and resistance to insulin action. The hypoglycemic response occurred during the late postprandial period.

analysis was performed using iodine labeled-swine insulin.

The levels of plasma glucose, insulin and C-peptide were determined before and two hours after each meal and at bedtime in the beginning of May (Table 2). The discrepancies between the levels of plasma glucose, insulin and Cpeptide indicated the existence of anti-insulin autoantibodies and insulin resistance. The fact that the patient complained of hypoglycemia occurring at lunch time and late evening around 16:00 o'clock corresponded to the low pre-meal plasma glucose level (Table 2: 12:00, 18:00). The hypoglycemic episodes appeared during the late postprandial period.

The free insulin to total insulin ratio ( $\mathrm{F} / \mathrm{T}$ ratio) was calculated in the plasma samples for the $75 \mathrm{gOGTT}$ performed in December 2009 (Table 3). The F/T ratio was significantly less than $100 \%$; therefore, we confirmed the existence of anti-insulin autoantibodies. Human leukocyte antigen (HLA) DRB1 typing showed a DNA sequencing pattern of DRB1* 0406*090102, DRB1 030201*030302, DQA1 030101*0302. A diagnosis of insulinoma was ruled out based on the find-
Table 3. 75gOGTT Performed in December, 2009; One Month after Termination of Adhesive Patch Form of LOXs

\begin{tabular}{crrrr}
\hline $\begin{array}{c}\text { Time } \\
(\mathrm{min})\end{array}$ & $\begin{array}{c}\text { PG } \\
(\mathrm{mg} / \mathrm{dL})\end{array}$ & \multicolumn{1}{c}{$\begin{array}{c}\text { Free IRI } \\
(\mu \mathrm{IU} / \mathrm{mL})\end{array}$} & $\begin{array}{c}\text { Total IRI } \\
(\mu \mathrm{IU} / \mathrm{mL})\end{array}$ & F/T ratio \\
\hline 0 & 89 & 7.7 & 96.5 & 8.0 \\
30 & 142 & 39.1 & 184.2 & 21.2 \\
60 & 199 & 64.6 & 244.5 & 26.4 \\
90 & 149 & 55.2 & 234.5 & 23.5 \\
120 & 139 & 85.0 & 290.1 & 29.3 \\
180 & 118 & 65.1 & 234.0 & 27.8 \\
\hline
\end{tabular}

HbA1c (NGSP) 5.2\%

On the plasma samples from 75gOGTT performed in December 2009 , free insulin to total insulin ratios (F/T ratio) was calculated. The $\mathrm{F} / \mathrm{T}$ ratio is much less than $100 \%$, so we can confirm the existence of anti-insulin antibody.

ings of abdominal ultrasound and computed tomography.

Considering the above findings, the patient was diagnosed with IAS.

As IAS is usually associated with medication use, all medications, including LOXs, were discontinued in the beginning of May, 2009. On the same day, the patient began taking an $\alpha$-glucosidase inhibitor (acarbose, $300 \mathrm{mg} /$ day) prior to each meal and continued this medication until August.

One month after the cessation of all medications, the patient was almost free from hypoglycemic episodes. Her glucose tolerance in June 2009 was normal (Table 1: One month after the first cessation of LOXs), and the titer of anti-insulin autoantibodies was decreased (Fig. 1). Accidentally, an adhesive patch of LOXs was re-prescribed by a neighboring clinic for shoulder pain in the beginning of October, 2009. In November, several hypoglycemic episodes recurred. The patient's glucose tolerance again deteriorated and the titer of anti-insulin autoantibodies again became ele- 


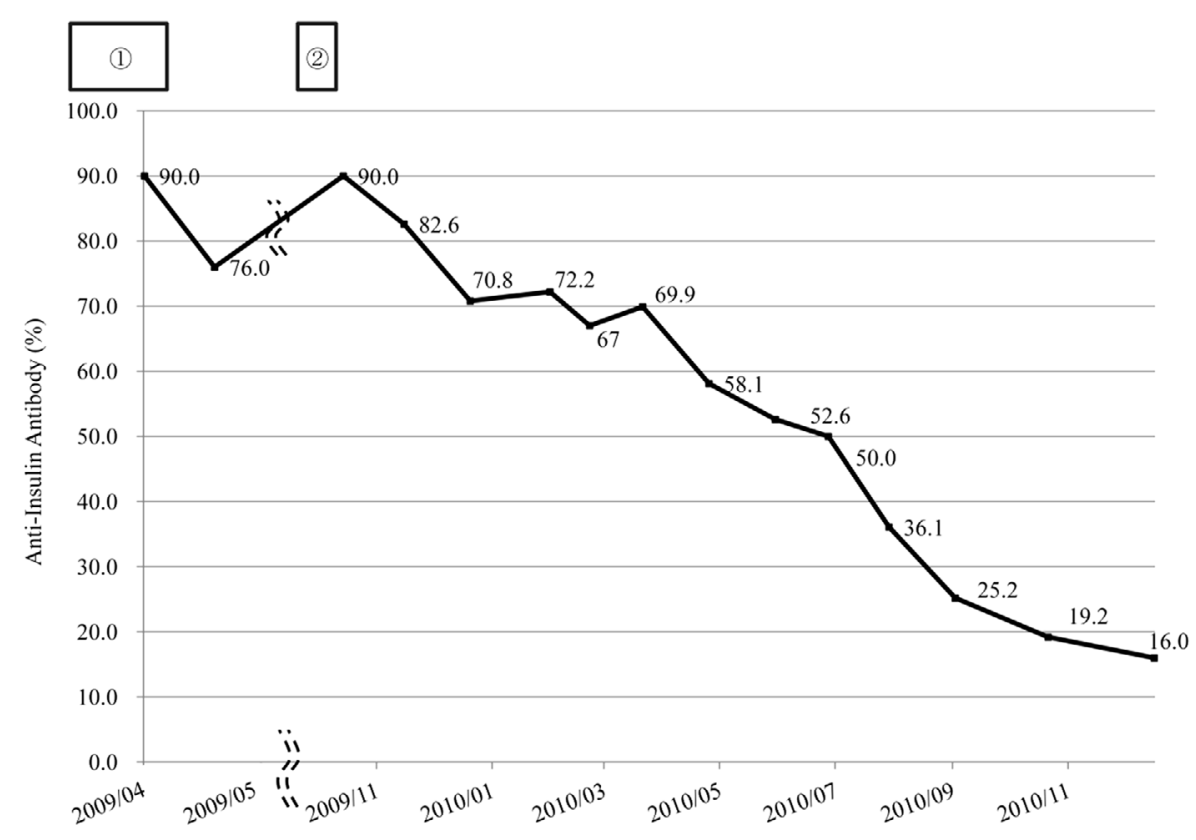

Figure 1. Clinical course of the titer of anti-insulin antibodies. The titers were high during LOXs periods, and decreased after the end of LOXs periods. (1)indicated the period while oral medicine of loxoprofen-sodium was administered. (2)indicated the period while adhesive patch form of LOXs was re-prescribed.

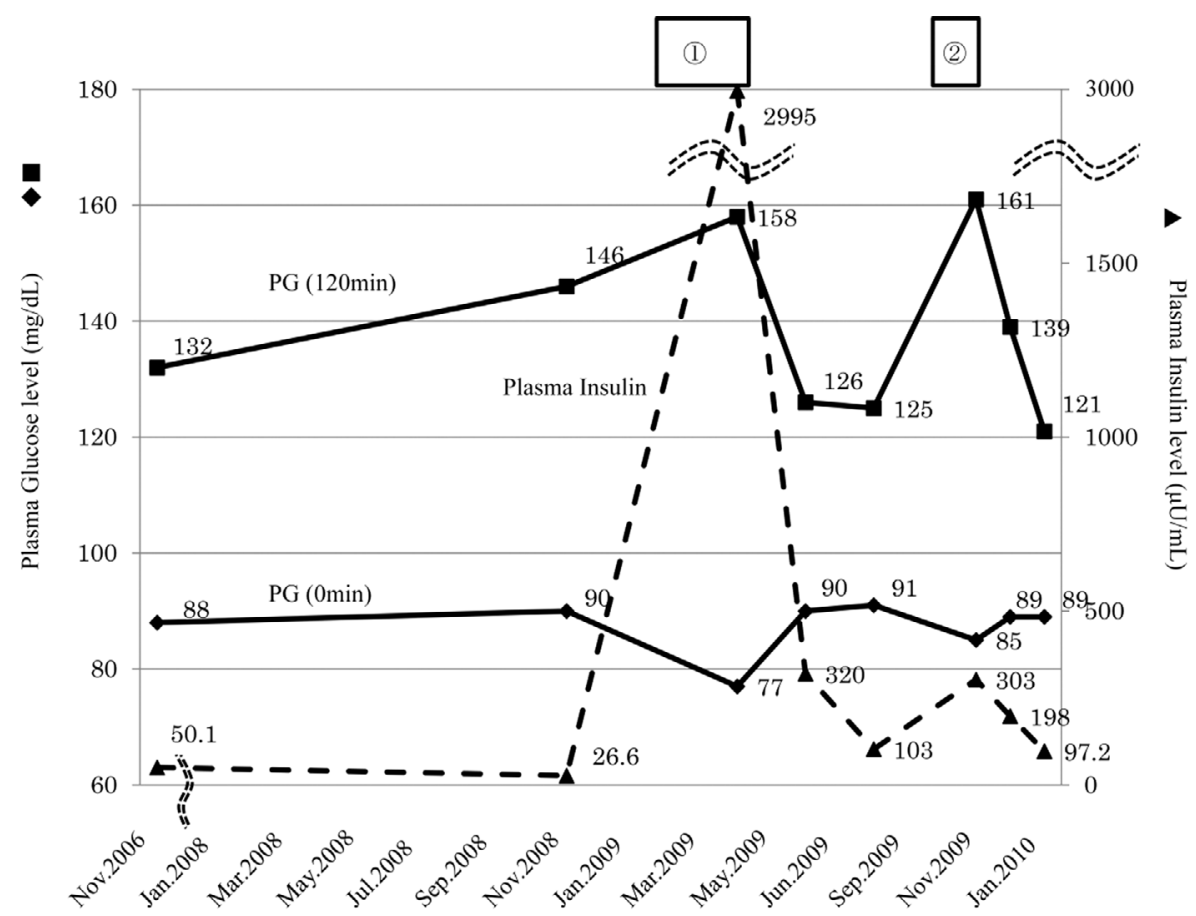

Figure 2. The plasma insulin $(120 \mathrm{~min})$ and glucose $(0 \mathrm{~min}, 120 \mathrm{~min})$ levels in repetitive $\mathbf{7 5} \mathrm{gOGTT}$. The plasma insulin and glucose levels in 75gOGTT were shown. The dashed line indicates plasma insulin level at $120 \mathrm{~min}$. The solid line; one indicated the plasma glucose at $0 \mathrm{~min}(\bullet)$, another at 120 min ( () . A broken line represents the change of plasma insulin level ( $\Delta$ ). Oral medication form of loxoprofen-sodium had been taken since February, 2009 until April, 2009; (1). And adhesive patch form of loxoprofen-sodium was accidentally re-administrated since October, 2009; (2).

vated (Fig. 2). The hypoglycemic symptoms and elevated anti-insulin autoantibodies recurred after the reuse of LOXs.

Following the discontinuation of the LOXs adhesive patch, the patient's hypoglycemic episodes completely disappeared. 75gOGTTs performed in December 2009 and January 2010 indicated that her glucose tolerance had im- 

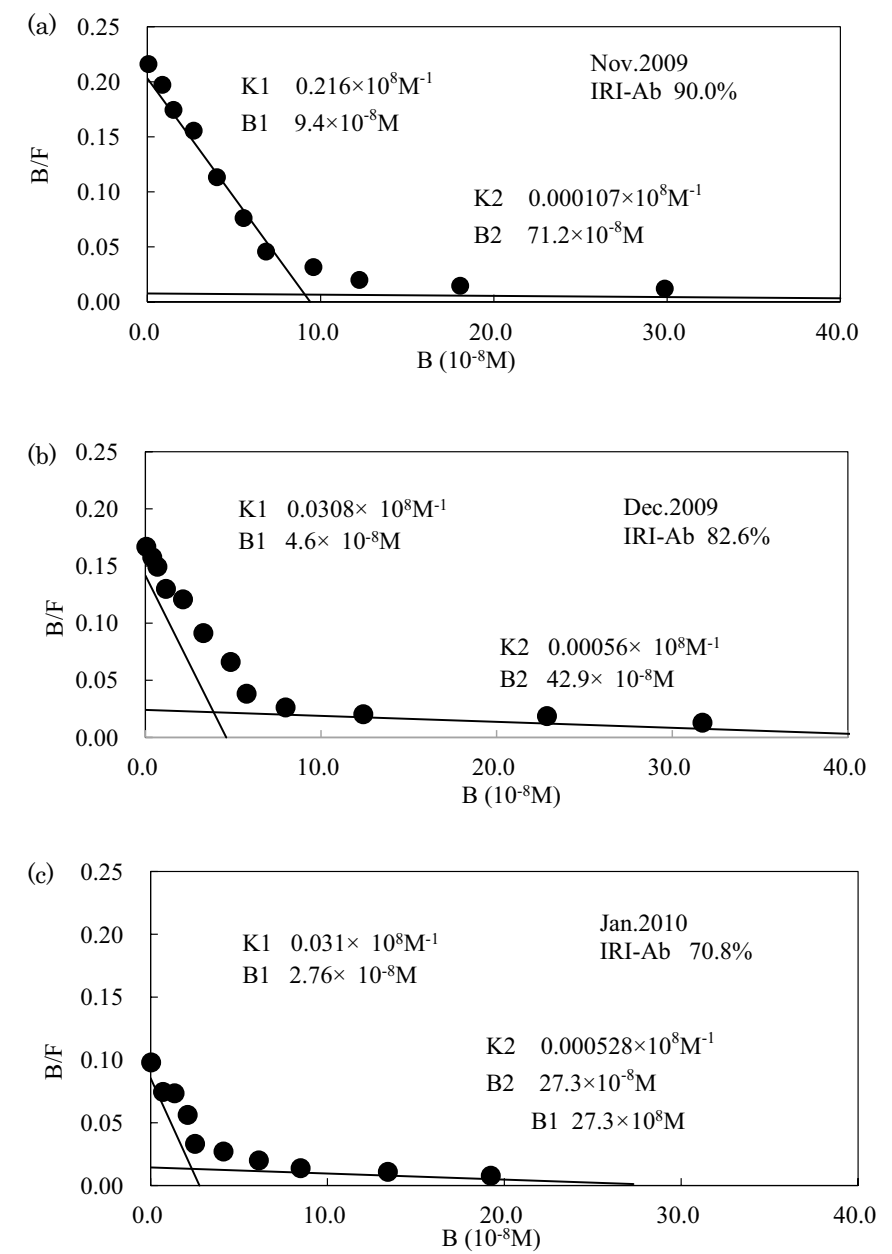

Figure 3. Scatchard plot analysis of insulin autoantibody in the patient. On Nov. 2009, the hypoglycemic attack recurred by an adhesive patch form of LOXs. Scatchard analysis was performed with ${ }^{125}$ I-human insulin and the serum of the patient (a). The same analysis was performed on Dec. 2009(b) and Jan. 2010(c), 1 month and 2 months after the cessation of LOXs, respectively. An affinity constant(K1, K2: excepting in January 2010) of the autoantibodies becomes higher, and a binding capacity(B1, B2) becomes lower in a row. B1 and B2 are binding capacities at the high-affinity and low-affinity sites, respectively. B/F: bound/free insulin ratio, B: bound insulin

proved (Fig. 2). A Scatchard analysis of anti-insulin autoantibodies was performed using ${ }^{125} \mathrm{I}$-human insulin and the patient's serum obtained in November and December 2009 and January 2010. The analysis revealed that the anti-insulin autoantibodies had a low affinity constant and a high binding capacity. The affinity constant (K1) of the anti-insulin autoantibodies decreased to approximately one-tenth and the binding capacity (B1) decreased to approximately one-third (Fig. 3). The quality of the anti-insulin autoantibodies appeared to change in association with decreases in the titer.

\section{Discussion}

Insulin autoimmune syndrome (IAS) is characterized by hypoglycemic episodes, an elevated plasma insulin level and positive anti-insulin autoantibodies without previous exposure to exogenous insulin. The first case of IAS was reported by Hirata et al. in 1970 (2). Most reported IAS cases have been related to medications containing the sulfhydryl
(SH) group (3). In addition, a strong association has been reported between IAS and HLA-DR4 (4). IAS is the third leading cause of spontaneous hypoglycemia in Japan (5). We herein described a case of IAS possibly triggered by LOXs, both in oral medication and adhesive patch form. To the best of our knowledge, only two cases of IAS related to LOXs have been reported $(6,7)$. Kishikawa et al. (6) reported the clinical course of a patient with a marked increase in the plasma insulin level without a history of insulin injection, a high titer of anti-insulin autoantibodies and positive drug-induced lymphocyte stimulation test (DLST) results. That patient did not possess the HLA-DR4, but rather the HLA-DR6, phenotype (6). Ninomiya et al. (7) reported the case of an IAS patient diagnosed based on the clinical course of a marked increase in the plasma insulin level without a history of insulin injection, a high titer of anti-insulin autoantibodies and the presence of the HLA DRB $1^{*} 0406$ phenotype, which is strikingly associated with IAS (4). However, their patient exhibited negative DLST re- 
sults (7)

In our case, the return of symptoms and re-elevation of the autoantibody titer following the accidental readministration of LOXs (Fig. 1) convinced us that LOXs was the cause of the IAS. DNA typing showed that our patient had HLA-DRB1 ${ }^{*} 0406$; however, the DLST results were negative. Our case indicated that IAS can be triggered by LOXs regardless of the form of medication and is not related to the existence of the SH group in the medication or its metabolites. In this case, positive DLST results were not needed to determine the causative drug for the development of IAS. In cases of IAS, the patient's medications should be examined carefully; adhesive patches can be easily overlooked. With the adhesive patch, the medication is significantly absorbed through the skin, thus provoking an immune reaction. If the medication does not have an $\mathrm{SH}$ group, then all medicines should be discontinued as soon as possible and a close follow-up of the course of symptoms should be provided.

The deterioration of the patient's glucose tolerance on 75 gOGTT was strongly correlated with the exacerbation of the IAS state (Table 1). The plasma glucose and insulin levels at 120 minutes of $75 \mathrm{gOGTT}$ reached their peaks during the LOXs periods (1) and (2) in Fig. 2) when the titer of antiinsulin autoantibodies also reached a peak of $90 \%$ (1) and (2) in Fig. 1). Following the discontinuation of LOXs, the titer decreased (Fig. 1), and the episodes of hypoglycemia disappeared. The plasma glucose level at 0 minutes of the $75 \mathrm{gOGTT}$ was a partial mirror image to that observed at 120 minutes (Fig. 2).

These findings can be explained by the characteristics of anti-insulin autoantibodies in patients with IAS. Y. Eguchi et al. reported that the autoantibodies can bind large amounts of insulin promptly and dissociate from the insulinautoantibody complex when the concentration of the serum insulin increases (8). Nasu et al. reported that the autoantibodies immediately bind to insulin secreted from the pancreas and mask the bioactivity of insulin in the early postprandial period, while the free insulin dissociated from the insulin-autoantibody complex exerts its intrinsic glucose lowering effects in the late period (9). Furthermore, in the Scatchard analysis in our case, the characteristics of the autoantibodies changed as the titer decreased. The values of $\mathrm{K} 1$ and $\mathrm{B} 1$ decreased in association with the progression of IAS (Fig. 3a, b, c). As the characteristics of the autoantibodies changed and the titer decreased, the patient's impaired glucose tolerance improved, the amount of insulin secreted from the pancreas decreased and the episodes of hypoglyce- mia disappeared.

The mechanism by which the discontinuation of LOXs reduces the titer and changes the characteristics of autoantibodies remains unclear. However, both the reduction of the titer and the changes in the characteristics affect the morbidity of patients with IAS.

We herein described the first case of IAS triggered by LOXs in both oral medication and adhesive patch form. Our cases indicates that clinicians should not ignore any medications, in any form, when seeking the causal agent of IAS.

\section{The authors state that they have no Conflict of Interest (COI).}

\section{Acknowledgement}

We would like to thank Dr. Atsushi Nakagawa, associate professor at the Department of Endocrinology, Kanazawa Medical University, for his valuable advice on the measurement of insulin autoantibodies.

\section{References}

1. http://www.jds.or.jp/jds_or_jp0/uploads/photos/818.pdf

2. Hirata $\mathrm{Y}$, Ishizu $\mathrm{H}$, Ouchi $\mathrm{H}$, et al. Insulin autoimmunity in a case of spontaneous hypoglycemia. Tonyobyo (Diabetes) 13: 312-320, 1970 (in Japanese).

3. Uchigata Y, Eguchi Y, Takayama-Hasumi S, Omori Y. Insulin autoimmune syndrome (Hirata Disease) : clinical features and epidemiology in Japan. Diabetes Res Clin Pract 22: 89-94, 1994.

4. Uchigata Y, Hirata Y, Omori Y, Iwamoto Y, Tokunaga K. Worldwide differences in the incidence of insulin autoimmune syndrome (Hirata disease) with respect to the evolution of HLA-DR4 alleles. Hum Immunol 61: 154-157, 2000.

5. Takayama-Hasumi S, Eguchi Y, Sato A, Morita C, Hirata Y. Insulin autoimmune syndrome is the third leading cause of spontaneous hypoglycemic attacks in Japan. Diabetes Res Clin Pract 10: 211-214, 1990.

6. Kishikawa H, Okada Y, Hirose A, et al. A case of insulin autoimmune syndrome associated with loxoprofen sodium. Tonyobyo (Diabetes) 47: 851-854, 2004 (in Japanese).

7. Ninomiya D, Hasebe S, Senba T, Sakai T, Uemura S. Assessment of the effect of $\alpha$-glucosidase inhibitor on a patient with insulin autoimmune syndrome by using continuous glucose monitoring: A case report. Tonyobyo (Diabetes) 55: 809-814, 2012 (in Japanese).

8. Eguchi Y. Scatchard analysis of insulin autoantibodies in the insulin autoimmune syndrome. Journal of Tokyo Women's Medical University 59: 1296-1305, 1998 (in Japannese. Abstract in English).

9. Nasu T, Suzuki R, Okamoto Y, et al. Late postprandial hypoglycemia due to bioactive insulin dissociation from autoantibody leading to unconsciousness in a patient with insulin autoimmune syndrome. Intern Med 50: 339-343, 2011.

(C) 2013 The Japanese Society of Internal Medicine

http://www.naika.or.jp/imonline/index.html 